\title{
EIGENVALUE ESTIMATES FOR HIGHER ORDER ELLIPTIC EQUATIONS*
}

\author{
HSU-TUNG KU, MEI-CHIN KU AND XIN-MIN ZHANG ${ }^{\dagger}$
}

\begin{abstract}
In this paper, we obtain good lower bound estimates of eigenvalues for various Dirichlet eigenvalue problems of higher order elliptic equations on bounded domains in $R^{n}$.
\end{abstract}

\section{Introduction}

Let $M$ be a bounded domain in the Euclidean $n$-space $R^{n}$ with smooth boundary $\partial M, n \geq 2$, and $\Delta$ be the Laplace operator defined by

$$
\triangle u=-\sum_{i=1}^{n} \frac{\partial^{2} u}{\partial x_{i}^{2}}, \quad u \in C^{2}(M) .
$$

Let $\left\{\tilde{\lambda}_{i}\right\}, 0<\tilde{\lambda}_{1} \leq \tilde{\lambda}_{2} \leq \cdots$, be the eigenvalues of the Dirichlet eigenvalue problem (a classical membrane problem)

$$
\begin{cases}\Delta u=\tilde{\lambda} u & \text { in } M \\ u=0 & \text { on } \partial M\end{cases}
$$

The Weyl's asymptotic formula asserts that

$$
\lim _{k \rightarrow \infty} k^{-2 / n} \tilde{\lambda}_{k}=C_{n}\left(\frac{1}{|M|}\right)^{2 / n}
$$

Received January 21,1994; revised May 25, 1993.

1991 Mathematics Subject Classification. Primary 35P15.

Key words and phrases. Dirichlet problem, generalized Polya conjecture, generalized ratio, eigenvalue, eigenfunction, consecutive eigenvalues, Fourier transform, mini-max principle, Rayleigh theorem.

†Partially supported by the University of South Alabama arts and science support and development fund. 
where $C_{n}=4 \pi^{2} /\left(w_{n}\right)^{2 / n}, w_{n}$ is the volume of the unit disk $R^{n}$ and $|M|$ denotes the volume of $M$. A consequence of (1.1) is the following (cf. [7]):

$$
\lim _{k \rightarrow \infty}\left(k^{-\frac{n+2}{n}} \sum_{i=1}^{k} \tilde{\lambda}_{i}\right)=\frac{n}{n+2} C_{n}\left(\frac{1}{|M|}\right)^{2 / n} .
$$

Thus, any good estimate of the eigenvalue $\tilde{\lambda}_{k}$ should be compatible with the Weyl's formula (1.1) or (1.2). The famous Polya conjecture states that

$$
\tilde{\lambda}_{k} \geq C_{n}\left(\frac{k}{|M|}\right)^{2 / n}, \quad k=1,2, \ldots
$$

This conjecture holds for some special domains in the plane [10]. In 1983 [8], Li and Yau verified that (1.3) holds in the average sense (cf. (1.2)), that is,

$$
\sum_{i=1}^{k} \tilde{\lambda}_{i} \geq \frac{n k}{n+2} C_{n}\left(\frac{k}{|M|}\right)^{2 / n}, \quad k=1,2, \ldots
$$

Now let $T(x)$ and $R(x)$ be polynomials of degrees $t$ and $r$ respectively with non-negative coefficients, say

$$
T(x)=\sum_{m=r+1}^{t} a_{m-r} x^{m}, a_{t-r}=1, \text { and } R(x)=\sum_{j=0}^{r} b_{j} x^{j} .
$$

In this paper we shall consider the following general eigenvalue problem:

$$
E(T, R): \begin{cases}T(\triangle) u=\mu(T, R) R(\triangle) u & \text { in } M, \\ \left(\frac{\partial}{\partial \nu}\right)^{s} u=0 & \text { on } \partial M, s=0,1, \cdots, t-1,\end{cases}
$$

with eigenvalues $\left\{\mu_{k}\right\}, 0<\mu_{1} \leq \mu_{2} \leq \cdots$, where $\mu_{k}=\mu_{k}(T, R)$, and $\frac{\partial}{\partial \nu}$ denotes the unit outward normal derivative on $\partial M$. Let $p \geq 0$ be a fixed integer, $p \leq r$. If $b_{j}=0$ for $j \leq p-1$ in $R(x)$, then $R(x)=R_{p}(x)$, where

$$
R_{p}(x)=\sum_{j=p}^{r} b_{j} x^{j}
$$

Hence, if we set

$$
\mu_{k, p}=\mu_{k}\left(T, R_{p}\right), k=1,2, \cdots,
$$

then

$$
\mu_{k}=\mu_{k, 0}, \text { and } \mu_{k, p}=\mu_{k, p-1}=\cdots=\mu_{k, 0} .
$$

We propose the following general eigenvalue estimates: 
Generalized Polya Conjecture. The eigenvalues $\left\{\mu_{k, p}\right\}$ satisfy the inequalities

$$
\mu_{k, p} \geq \frac{1}{b}\left\{\sum_{m=1}^{t-r} a_{m}\left(C_{n}\right)^{m}\left(\frac{k}{|M|}\right)^{2 m / n}\right\}^{(t-r) /(t-p)}, k=1,2, \cdots,
$$

where $b=R_{p}(1)$.

Set

$$
Q_{k, p}(M)=\frac{1}{b}\left\{\sum_{m=1}^{t-r} \frac{n a_{m}}{n+2 m}\left(C_{n}\right)^{m}\left(\frac{k}{|M|}\right)^{2 m / n}\right\}^{(t-r) /(t-p)},
$$

and

$$
\lambda_{k, p}=\mu_{k}\left(T, x^{p}\right), k=1,2, \cdots,
$$

We shall verify that the Generalized Polya Conjecture holds in the average sense for many domains $M$ in $R^{n}$. More precisely we have

Theorem A.Let $\alpha$ and $\beta$ be any positive real numbers and $\lambda_{1, p} \geq 1$. Then

i) $\sum_{i=1}^{k} \mu_{i, p}^{\alpha} \geq k\left\{Q_{k, p}(M)\right\}^{\alpha}, k=1,2, \cdots \cdots$

ii) $\sum_{i=1}^{k} \frac{1}{\mu_{i, p}^{\alpha}} \leq k\left\{Q_{k, p}(M)\right\}^{-\alpha}, k=1,2, \cdots$.

iii) $\frac{\sum_{i=1}^{k} \mu_{i, p}^{\alpha}}{\sum_{i=1}^{k} \mu_{i, p}^{-\beta}} \geq\left\{Q_{k, p}(M)\right\}^{\alpha+\beta}, k=1,2, \cdots$.

The estimates of $\mu_{k, p}$ for some special cases are also contained in [3] [4] [6] [7].

The generalized ratio of the consecutive eigenvalues $\mu_{k+1, p}$ and $\mu_{k, p}$ is defined as the quotient $\mu_{k+1, p} / \mu_{k, p}^{t /(t-r)}$. We have the following interesting estimate of the upper bounds of the generalized ratio which is independent of the integer $k$.

Theorem B. If $\eta_{1, p} \geq 1$, then

$$
\frac{\mu_{k+1, p}}{\mu_{k, p}^{t /(t-r)}} \leq b^{r /(t-r)}\left\{1+\frac{4}{n^{2}} \sum_{m=r+1}^{t} m(n+2 m-2) a_{m-r}\right\},
$$

where $\eta_{k, p}=\mu_{k}\left(x^{p+1}, x^{p}\right)$.

The hypotheses $\lambda_{1, p} \geq 1$ and $\eta_{1, p} \geq 1$ in Theorems $A$ and $B$ respectively are unnecessary if $\mu_{i, p}=\lambda_{i, p}$, i.e. $R(x)=R_{p}(x)$. As an immediate corollary of Theorem $\mathrm{B}$ we obtain the following inequality:

$$
\frac{\eta_{k+1, p}}{\eta_{k, p}^{p+1}} \leq 1+\frac{4}{n^{2}}(p+1)(n+2 p)
$$


This generalizes the following inequality of Payne, Polya and Weinberger [10] because $\eta_{k+1,0}=\widetilde{\lambda}_{k+1}$ and $\eta_{k, 0}=\widetilde{\lambda}_{k}$ :

$$
\frac{\tilde{\lambda}_{k+1}}{\widetilde{\lambda}_{k}} \leq 1+\frac{4}{n}
$$

\section{Eigenvalue Estimates}

For any fixed integer $p, 0 \leq p \leq r$, set

$$
<u, v>=\int_{M} u(x) \Delta^{p} v(x) d x .
$$

A function $u$ is said to satisfy the condition $(* m)$ if

$$
\left(\frac{\partial}{\partial \nu}\right)^{s} u=0 \text { on } \partial M \text { for } s=0,1, \cdots, m-1 .
$$

Suppose the function $u$ satisfies $(* t)$ and $\langle u, u\rangle=1$. Then we can verify the following inequality (cf.[3]):

$$
<u, \triangle^{m} u>\leq<u, \triangle^{m+1} u>^{m /(m+1)}, 1 \leq m \leq t-p .
$$

Lemma 2.1 (cf. [3]) For $k \geq 1$, we have

$$
\lambda_{k, r} \geq \sum_{m=r+1}^{t} a_{m-r}\left(\eta_{k, r}\right)^{m-r}
$$

Thus $\lambda_{k, r} \geq 1$ if $\eta_{1, r} \geq 1$.

Proof. The inequality (2.2) follows easily from (2.1), maxi-mini principle and the Rayleigh Theorem.

Lemma 2.2 (cf. [3]) For any $k=1,2, \cdots$,

i) $\mu_{k, p} \leq \frac{1}{b} \lambda_{k, p}$ if $\eta_{1, p} \geq 1$

ii) $\mu_{k, p} \geq \frac{1}{b}\left(\lambda_{k, p}\right)^{(t-r) /(t-p)}$ if $\lambda_{k, p} \geq 1$.

Proof. Let

$$
(u, v)=\int_{M} u(x) R_{p}(\Delta) v(x) d x .
$$

and $\left\{u_{i}\right\}_{1}^{k}$ (resp. $\left\{v_{i}\right\}_{1}^{k}$ ) be a set of orthonormal eigenfunctions associated with the eigenvalues $\left\{\lambda_{i, p}\right\}_{1}^{k}$ (resp. $\left.\left\{\mu_{i, p}\right\}_{1}^{k}\right)$. By maxi-mini method, there exists $v=\sum_{j=1}^{k} c_{j} u_{j}$ such that $\left(v, v_{i}\right)=0$ for $i=1,2, \cdots, k-1$, and $\langle v, v\rangle=1$. From (2.1) we have

$$
(<v, \Delta v>)^{j-p} \leq<v, \Delta^{j-p} v>, j=p+1, \cdots, r
$$


and (2.3) also holds for $j=p$ because $1=\langle v, v\rangle$. Moreover from Rayleigh Theorem and hypothesis we obtain

$$
<v, \Delta v>=\int_{M} v \Delta^{p+1} v \geq \eta_{1, p} \geq 1
$$

Hence it follows from (2.3) and (2.4) that $(v, v) \geq b$, and so,

$$
\mu_{k, p} \leq \frac{\int_{M} v T(\Delta) v}{(v, v)} \leq \frac{\sum_{j=1}^{k} c_{i} c_{j} \int_{M} u_{i} T(\Delta) u_{j}}{b} \leq \frac{\sum_{j=1}^{k} c_{j}^{2} \lambda_{j, p}}{b} \leq \frac{1}{b} \lambda_{k, p}
$$

because $\sum_{j=1}^{k} c_{j}^{2}=1$. This proves (i). The proof of (ii) is similar.

If $R(x)=R_{p}(x)=x^{p}$ and $\mu_{k, p}=\lambda_{k, p}$, Lemma 2.2 holds trivially. Hence the assumptions $\eta_{1, p} \geq 1$ and $\lambda_{1, p} \geq 1$ are unnecessary. Observe that (ii) also holds under the hypothesis $\eta_{1,0} \geq 1$ by Lemma 2.1 .

Lemma 2.3 If $\eta_{1,0} \geq 1$, then

$$
\lambda_{k, p} \leq \lambda_{k, 0} \leq\left(\lambda_{k, p}\right)^{t /(t-p)} .
$$

Proof. Let $R_{p}(x)=x^{p}$. Then $\lambda_{k, p}=\mu_{k, p}$. Notice that $b=R_{p}(1)=1$. Since $\eta_{1,0} \geq 1$, by Lemma 2.2 we have

$$
\mu_{k, 0} \leq \lambda_{k, 0} \leq\left(\mu_{k, 0}\right)^{t /(t-p)} .
$$

But $\mu_{k, p}=\mu_{k, 0}$ by (1.5). Hence $\mu_{k, 0}=\lambda_{k, p}$. This proves the Lemma by (2.5).

Proof of Theorem A. Let

$$
P_{k, p}(M)=\sum_{m=1}^{t-r} \frac{n a_{m}}{n+2 m}\left(C_{n}\right)^{m}\left(\frac{k}{|M|}\right)^{2 m / n} .
$$

It was proved in [4] (and [6] for $p$ even) that

$$
\sum_{i=1}^{k} \lambda_{i, p} \geq k P_{k, p}(M), k=1,2, \cdots
$$

Hence we can show as in [6] using [4], that for any positive real number $d>0$, we have

$$
\sum_{i=1}^{k} \lambda_{i, p}^{d} \geq k\left\{P_{k, p}(M)\right\}^{d}, k=1,2, \cdots
$$

and

$$
\sum_{i=1}^{k} \frac{1}{\lambda_{i, p}^{d}} \leq k\left\{P_{k, p}(M)\right\}^{-d}, k=1,2, \cdots
$$


i) Since $\lambda_{i, p} \geq 1$, it follows from Lemma 2.2 that $\mu_{i, p} \geq \frac{1}{b}\left(\lambda_{i, p}\right)^{(t-r /(t-p)}$, hence by (2.7), we obtain

$$
\begin{aligned}
\sum_{i=1}^{k} \mu_{i, p}^{\alpha} & \geq \frac{1}{b^{\alpha}} \sum_{i=1}^{k}\left(\lambda_{i, p}\right)^{\alpha(t-r) /(t-p)} \geq \frac{k}{b^{\alpha}}\left\{P_{k, p}(M)\right\}^{\alpha(t-r) /(t-p)} \\
& =k\left\{Q_{k, p}(M)\right\}^{\alpha} .
\end{aligned}
$$

ii) Since $\lambda_{1, p} \geq 1$, again by Lemma 2.2 we have

$$
\frac{1}{\mu_{i, p}^{\alpha}} \leq \frac{b^{\alpha}}{\lambda_{i, p}^{\alpha(t-r) /(t-p)}}
$$

hence by (2.7),

$$
\sum_{i=1}^{k} \frac{1}{\mu_{i, p}^{\alpha}} \leq b^{\alpha} \sum_{i=1}^{k} \frac{1}{\lambda_{i, p}^{\alpha(t-r) /(t-p)}} \leq k b^{\alpha}\left\{P_{k, p}(M)\right\}^{-\alpha(t-r) /(t-p)}=k\left\{Q_{k, p}(M)\right\}^{-\alpha} .
$$

iii)

$$
\begin{aligned}
\sum_{i=1}^{k} \mu_{i, p}^{\alpha} & \geq k\left\{Q_{k, p}(M)\right\}^{\alpha}=k\left\{Q_{k, p}(M)\right\}^{-\beta}\left\{Q_{k, p}(M)\right\}^{\alpha+\beta} \\
& \geq\left\{\sum_{i=1}^{k} \frac{1}{\mu_{i, p}^{\beta}}\right\}\left\{Q_{k, p}(M)\right\}^{\alpha+\beta} \text { by }(\mathrm{ii}) .
\end{aligned}
$$

from which the desired inequality follows.

As a corollary of Theorem A we have:

Theorem 2.4. Let $0 \leq p \leq r$ be as above, and let $\lambda_{1, p} \geq 1$. Then for $k=1,2, \cdots$,

$$
\sum_{i=1}^{k} \mu_{i, p} \geq \frac{k}{b} \sum_{m=1}^{t-r} a_{m}\left\{\frac{n}{n+2 m}\left(C_{n}\right)^{m}\left(\frac{k}{|M|}\right)^{2 m / n}\right\}^{m /(m+r-p)} .
$$

Proof. Set

$$
\zeta_{k, m+r}=\mu_{k}\left(x^{m+r}, R_{p}\right), 1 \leq m \leq t-r .
$$

Theorem A implies

$$
\sum_{i=1}^{k} \zeta_{i, m+r} \geq \frac{k}{b}\left\{\frac{n}{n+2 m}\left(C_{n}\right)^{m}\left(\frac{k}{|M|}\right)^{2 m / n}\right\}^{m /(m+r-p)} .
$$

Moreover, from [3, Theorem 7] we have

$$
\sum_{i=1}^{k} \mu_{i, p} \geq \sum_{i=1}^{k} \sum_{m=1}^{t-r} a_{m} \zeta_{i, m+r}
$$


Thus, we establish the inequality (2.9) using (2.10) and (2.11).

Now let

$$
L u=(-1)^{t} \sum_{|\alpha|=2 t} c_{\alpha} D^{\alpha} u \quad\left(c_{\alpha} \text { 's are constants }\right)
$$

be an ellipitic differential operator, that is, there exists a constant $c>0$ such that

$$
\sum_{|\alpha|=2 t} c_{\alpha} \xi^{\alpha} \geq c|\xi|^{2 t}, \text { for all } \xi=\left(\xi_{1}, \cdots, \xi_{n}\right) \in R^{n}
$$

Here $\alpha=\left(\alpha_{1}, \cdots, \alpha_{n}\right)$ is an $n$-tuple of nonnegative integers; $|\alpha|=\alpha_{1}+\cdots+\alpha_{n}$, and $D^{\alpha}=D_{1}^{\alpha_{1}} \cdots D_{n}^{\alpha_{n}}, D_{j}=\partial / \partial x_{j}$. Let $\Lambda_{k, p}$ 's be the eigenvalues of the Dirichlet problem (where $0 \leq p \leq r<t$ ):

$$
\left\{\begin{array}{l}
L_{u}=\Lambda R_{p}(\triangle) u \quad \text { in } M \\
u \text { satisfies }(* t)
\end{array}\right.
$$

Theorem 2.5. For $k=1,2, \cdots$, we have

i) $\Lambda_{k, t} \geq c \zeta_{k, t}$,

and

ii) $\sum_{j=1}^{k} \Lambda_{j, t} \geq \frac{c k}{b}\left\{\frac{n}{n+2(t-r)}\left(\frac{k}{|M|}\right)^{2(t-r) / n}\right\}^{(t-r) /(t-p)}$.

Proof. Let $\left\{w_{i}\right\}_{1}^{k}$ be the orthonormal eigenfunctions corresponding to the eigenvalues $\left\{\zeta_{i, t}\right\}_{1}^{k}$ and let $w \in C^{2 t}(M)$ be an non-zero function satisfying $(* t)$ and $\left(w, w_{j}\right)=$ $0, j=1,2, \cdots, k-1$. Since $w \mid \partial M=0$, we can extend $w$ to $R^{n}$ by letting $w(x)=0$ for $x \in R^{n} \backslash M$. Let $\widehat{w}(x)$ denote the Fourier transform of $w(x)$. Then

$$
\left(\widehat{\frac{\partial}{\partial x_{j}}} w\right)(\xi)=i \xi_{j} \widehat{w}(\xi), \quad i=\sqrt{-1}
$$

hence

$$
\widehat{D^{\beta} w}(\xi)=i^{|\beta|} \xi^{\beta} \widehat{w}(\xi)
$$

Moreover, for any $h \geq 0$,

$$
\begin{gathered}
\left|\nabla \widehat{\triangle^{h} w}(\xi)\right|=|\xi|^{2 h+1}|\widehat{w}(\xi)|, \\
\left.\left|\widehat{\Delta^{h} w}(\xi)=\right| \xi\right|^{2 h}|\widehat{w}(\xi)| .
\end{gathered}
$$

Write $D^{\alpha} w=D^{\beta+\gamma} w$ with $|\beta|=|\gamma|=t$. By integration by parts and the Planchel 
Theorem we have

$$
\begin{aligned}
(-1)^{t} \int_{M} w(x) D^{\alpha} w(x) d x & =\int_{M}\left(D^{\beta} w\right)\left(D^{\alpha} w\right) d x \\
& =\frac{1}{4} \int_{R^{n}}\left\{\left[D^{\beta} w+D^{\gamma} w\right]^{2}-\left[D^{\beta} w-D^{\gamma} w\right]^{2}\right\} d x \\
& =\frac{1}{4} \int_{R^{n}}\left\{\left|\widehat{D^{\beta} w}+\widehat{D^{\gamma} w}\right|^{2}-\left|\widehat{D^{\beta} w}-\widehat{D^{\gamma} w}\right|^{2}\right\} d \xi \\
& =\frac{1}{2} \int_{M}\left(\widehat{D^{\beta} w} \widehat{\widehat{D^{\gamma} w}}+\widehat{\widehat{D^{\beta} w}} \widehat{D^{\gamma} w}\right) d \xi \\
& =\operatorname{Re} \int_{M} \widehat{D^{\beta} w \widehat{D^{\gamma} w}} d \xi \quad(\mathrm{by}(2.15)) \\
& =\int_{M} \xi^{\beta+\gamma}|\widehat{w}(\xi)|^{2} d \xi \quad \\
& =\int_{M} \xi^{\alpha}|\widehat{w}(\xi)|^{2} d \xi
\end{aligned}
$$

Observe that

$$
\int_{M}|\xi|^{2 t}|\widehat{w}(\xi)|^{2} d \xi=\int_{M} w \Delta^{t} w d x
$$

For instance, if $t=2 h$, then

$$
\begin{array}{rlr}
\int_{M}|\xi|^{2 t}|\widehat{w}(\xi)|^{2} d \xi & =\int_{M}\left(|\xi|^{2 h}|\widehat{w}(\xi)|\right)^{2} d \xi \\
& =\int_{M}\left|\widehat{\Delta^{h} w}(\xi)\right|^{2} d \xi \quad \text { (by (2.14)) } \\
& =\int_{M}\left|\triangle^{h} w(x)\right|^{2} d x \quad \text { (by Planchel Theorem) } \\
& =\int_{M} w \Delta^{t} w d x . \quad \text { (by Green's Theorem) }
\end{array}
$$

It follows that

$$
\begin{aligned}
\int_{M} w L w d x & =\int_{M} \sum_{|\alpha|=2 t} c_{\alpha} \xi^{\alpha}|\widehat{w}(\xi)|^{2} d \xi \\
& \geq c \int_{M}|\xi|^{2 t}|\widehat{w}(\xi)|^{2} d \xi \quad(\operatorname{by}(2.12)) \\
& =c \int_{M} w \triangle^{t} w d x .
\end{aligned}
$$

From the mini-max principle we have

$$
\Lambda_{k, t}=\inf _{E} \sup \left\{\left.\frac{\int_{M} w L w}{(w, w)}\right|^{0 \neq w \in C^{2 t}(M), w \text { satisfies }(* t)} \begin{array}{c} 
\\
E \subset C^{2 t}(M), \operatorname{dim} E=k
\end{array}\right\},
$$


and

$$
\zeta_{k, t}=\inf _{E} \sup \left\{\frac{\int_{M} w \Delta^{t} w}{(w, w)} \mid \begin{array}{c}
0 \neq w \in C^{2 t}(M), w \text { satisfies }(* t) \\
E \subset C^{2 t}(M), \operatorname{dim} E=k
\end{array}\right\} .
$$

This implies (2.13) by (2.18). Thus, the inequality (2.14) follows from theorem $\mathrm{A}$ and (2.13).

We conjecture that Theorem 2.5 remains true for the differential operator $\widetilde{L}$ which is self-adjoint and uniformly elliptic, that is, it satisfies (2.12) and $c_{\alpha}^{\prime} \mathrm{s}$ are functions $c_{\alpha}(x) \in C^{t}(\bar{M})$ and there is a constant $K>0$ such that

$$
\sum_{\alpha}\left|c_{\alpha}(x)\right|^{2} \leq K, \text { for all } x \in M
$$

We shall show that this conjecture is true for $t=1$. Since $\tilde{L}$ is self-adjoint, we may assume that $\widetilde{L}$ is in the divergent form, that is,

$$
\widetilde{L} u=-\sum_{i, j} D_{i}\left(c_{i j}(x) D_{j} u\right)
$$

where the matrix $\left(c_{i j}(x)\right)$ is symmetric. Let $\left\{\widetilde{\Lambda}_{k}\right\}$ be the eigenvalues of the eigenvalue problem:

$$
\begin{cases}\widetilde{L} u=\widetilde{\Lambda} u & \text { in } M \\ u=0 & \text { on } \partial M .\end{cases}
$$

The following result generalizes the result of $\mathrm{Li}$ and $\mathrm{Yau}$ (1.4).

\section{Theorem 2.6.}

i) $\tilde{\Lambda}_{k} \geq c \widetilde{\lambda}_{k}, k=1,2, \cdots$,

and

ii) $\sum_{j=1}^{k} \widetilde{\Lambda}_{j} \geq \frac{c k n}{n+2} C_{n}\left(\frac{k}{|M|}\right)^{2 / n}, k=1,2, \cdots$.

Proof. Let $\left\{\varphi_{k}\right\}$ be the orthonormal eigenfunctions corresponding to $\left\{\widetilde{\Lambda}_{k}\right\}$. Since the quadratic form

$$
Q(w)=\int_{M} w \widetilde{L} w
$$

satisfies $Q\left(\varphi_{k}\right)=\widetilde{\Lambda}_{k}=\tilde{\Lambda}_{k}<\varphi_{k}, \varphi_{k}>$, hence the mini-max principle also holds for the eigenvalue problem (2.19). Thus,

$$
\tilde{\Lambda}_{k}=\inf _{E} \sup \left\{\frac{\int_{M} w \tilde{L} w}{\langle w, w\rangle} \mid \begin{array}{c}
0 \neq w \in C^{2}(M), w \mid \partial M=0 \\
E \subset C^{2}(M), \operatorname{dim} E=k .
\end{array}\right\}
$$

Nuw for any $w \in C^{2}(M), w \neq 0$ and $w \mid \partial M=0$,

$$
\begin{aligned}
\int_{M} w \widetilde{L} w & =\int_{M} \sum_{i, j} c_{i, j}(x) D_{i} w D_{j} w \geq c \int_{M}|\nabla w|^{2} \\
& =c \int_{M} w \Delta w .
\end{aligned}
$$


Hence it follows from the mini-max principle that we have the inequality (2.20). Thus, we can establish the inequality (2.21) via the estimate (1.4).

To conclude this section we verify the following comparison theorem for eigenvalus $\zeta_{k, t}$ 's.

Theorem 2.7. Let $0 \leq p \leq r<m \leq t$, and $b_{p}=1$ in $R_{p}(x)$. Then we have

i) $\zeta_{k, t} \geq\left(\zeta_{k, t}\right)^{t / m}, \quad k=1,2, \cdots$;

ii) $\sum_{i=1}^{k}\left(\zeta_{k, t}\right)^{s} \geq k^{(m-t) / t}\left\{\sum_{i=1}^{k}\left(\zeta_{i, m}\right)^{s}\right\}^{t / m}, k=1,2, \cdots$, and $s>0$ is any real number.

Proof. i) Let $W_{k}$ be the subspace of $L^{2}(M)$ generated by $w_{1}, w_{2}, \cdots, w_{k}$, and so $\left(w_{i}, w_{j}\right)=\delta_{i, j}$. Let $w=\sum_{j=1}^{k} a_{j} w_{j} \in W_{k}$ and $\int_{M} w \Delta^{p} w=1$. Then

$$
\sum_{j=1}^{k} a_{j}^{2}=(w, w) \geq \int_{M} w \triangle^{p} w=1
$$

hence

$$
\frac{\int_{M} w \Delta^{m} w}{(w, w)} \leq \frac{\left(\int_{M} w \Delta^{t} w\right)^{m / t}}{(w, w)} \leq\left(\sum_{j=1}^{k} a_{j}^{2}\right)^{(m-t) / t} \quad\left(\zeta_{k, t}\right)^{m / t} \leq\left(\zeta_{k, t}\right)^{m / t} .
$$

Now for $u \neq 0, u \in W_{k}$, let $\|u\|^{2}=\int_{M} u \triangle^{p} u$ and set $w=u /\|u\|$. Then $\int_{M} w \triangle^{p} w=1$ and

$$
\frac{\int_{M} u \Delta^{m} u}{(u, u)}=\frac{\int_{M} w \Delta^{m} w}{(w, w)}
$$

Since $w_{i}$ 's also satisfy $(* m)$; hence (i) follows from the mini-max principle, $(2.22)$ and (2.23).

ii) By (i) and Hölder's inequality.

The special case of Theorem 2.7 (i) when $p=r=0$ was proved in [3] and [2] (for $m=k=1)$.

\section{Generalized Ratio}

Theorem 3.1. If $\eta_{1, p} \geq 1$, then

$$
\mu_{k+1, p}-b^{\frac{r}{t-r}} \mu_{k, p}^{\frac{t}{t-r}} \leq \frac{4}{b k n^{2}} \sum_{m=r+1}^{t} m(n+2 m-2) a_{m-r}\left\{\sum_{i=1}^{k}\left(b \mu_{i, p}\right)^{\frac{t}{t-r}}\right\}^{\frac{m}{t}} k^{\frac{t-m}{t}} .
$$

Proof. The following result is proved in [1]. The special case $L=\Delta$ was obtained by Payne, Polya and Weinberger in [10].

$$
\lambda_{k+1,0}-\lambda_{k, 0} \leq \frac{4}{k n^{2}} \cdot \sum_{m=r+1}^{t} m(n+2 m-2) a_{m-r}\left\{\sum_{i=1}^{k} \lambda_{i, 0}\right\}^{m / t} k^{(t-m) / t} .
$$


Thus, it follows from Lemmas 2.2 and 2.3 that

$$
\begin{aligned}
\mu_{k+1, p} & \leq \frac{1}{b} \lambda_{k+1, p} \leq \frac{1}{b}\left(\lambda_{k+1,0}-\lambda_{k, 0}\right)+\frac{1}{b} \lambda_{k, 0} \\
& \leq \frac{4}{b k n^{2}} \sum_{m=r+1}^{t} m(n+2 m-2) a_{m-r}\left\{\sum_{i=1}^{k} \lambda_{i, 0}\right\}^{m / t} k^{\frac{t-m}{t}}+\frac{1}{b} \lambda_{k, 0}(\text { by }(3.1)) \\
& \leq \frac{4}{b k n^{2}} \sum_{m=r+1}^{t} m(n+2 m-2) a_{m-r}\left\{\sum_{i=1}^{k} \lambda_{i, p}^{t /(t-p)}\right\}^{m / t} k^{\frac{t-m}{t}}+\frac{1}{b} \lambda_{k, p}^{\frac{t}{t-p}} \\
& \leq \frac{4}{b k n^{2}} \sum_{m=r+1}^{t} m(n+2 m-2) a_{m-r}\left\{\sum_{i=1}^{k}\left(b \mu_{i, p}\right)^{t /(t-r)}\right\}^{m / t} k^{\frac{t-m}{t}}+b^{\frac{r}{t-r}} \mu_{k, p}^{\frac{t}{t-r}}
\end{aligned}
$$

This establishes the Theorem.

Proof of Theorem B. Since $\lambda_{k, 0} \geq 1$, form the proof of Theorem 3.1, we get

$$
\begin{aligned}
\mu_{k+1, p} & \leq \frac{4}{b k n^{2}} \sum_{m=r+1}^{k} m(n+2 m-2) a_{m-r}\left\{k \lambda_{k, 0}\right\}^{m / t} k^{\frac{t-m}{t}}+\frac{1}{b} \lambda_{k, 0} \\
& \leq \frac{1}{b}\left\{1+\frac{4}{n^{2}} \sum_{m=r+1}^{t} m(n+2 m-2) a_{m-r}\right\} \lambda_{k, p}^{t /(t-p)} \quad \text { (by Lemma 2.3) } \\
& \leq \frac{1}{b}\left\{1+\frac{4}{n^{2}} \sum_{m=r+1}^{t} m(n+2 m-2) a_{m-r}\right\}\left(b \mu_{k, p}\right)^{\frac{t}{t-r}} \quad \text { (by Lemma 2.2) } \\
& =b^{r /(t-r)}\left\{1+\frac{4}{n^{2}} \sum_{m=r+1}^{t} m(n+2 m-2) a_{m-r}\right\} \mu_{k, p}^{t /(t-r)}
\end{aligned}
$$

This concludes the proof.

From [5], we also have the following inequality:

$$
\lambda_{k+1,1}-\lambda_{k, 1} \leq \frac{4}{k(n+2)^{2}} \sum_{m=1}^{t-r}(m+1)(n+2 m) a_{m}\left\{\sum_{i=1}^{k} \lambda_{i, 1}\right\}^{\frac{n_{b}}{i-1}} k^{\frac{i-m-1}{i-1}} .
$$

By repeating the arguments above using this inequality we have

Theorem 3.2. If $\eta_{1, p} \geq 1$, then

i)

$$
\begin{aligned}
& \mu_{r+1, p}-b^{r /(t-r)} \mu_{k, p}^{t /(t-r)} \\
& \leq \frac{4}{b(n+2)^{2} k} \sum_{m=1}^{t-r}(m+1)(n+2 m) a_{m}\left\{\sum_{i=1}^{k}\left(b \mu_{i, p}\right)^{\frac{t}{t-r}}\right\}^{\frac{m}{t-1}} k^{\frac{t-m-1}{t-1}} .
\end{aligned}
$$


ii)

$$
\frac{\mu_{k+1, p}}{\mu_{k, p}^{t /(t-r)}} \leq b^{r /(t-r)}\left\{1+\frac{4}{(n+2)^{2}} \sum_{m=1}^{t-r}(m+1)(n+2 m) a_{m}\right\} .
$$

As a special case if $\eta_{1, p} \geq 1$, we have

$$
\frac{\eta_{k+1, p}}{\eta_{k, p}^{p+1}} \leq 1+\frac{8}{n+2}
$$

This inequality is much sharper than the one in (1.10) if $p \geq 1$.

Acknowledgement. It is our pleasure to thank referee for his suggestion of rearranging this paper.

\section{References}

[1] Z.C. Chen and C.L. Qian, "Estimates for discrete spectrum of Laplace operator with any order," Jour. China Univ. of Sci. and Tech. 20(1990), 259-268.

[2] Y.G. Gu, "Some notes on eigenvalue problems of elliptic equations of higher order," Jour. of Sys. Sci. and Math. Sci, 9(4)(1989), 313-320.

[3] S.M. Hook, "Inequalities for eigenvalues of self-adjoint operators," Trans. Amer. Math. Soc., 318(1990), 237-259.

[4] H.T. Ku and M.C. Ku, "Eigenvalue Estimates and The Generalized Polya Conjecture," Bull. Inst. Math. Academia, Sinica, Vol. 19, No. 4(1991) 289-295.

[5] H.T. Ku and M.C. Ku, "The difference of consecutive eigenvalues," Jour. of Australian Math. Soc., to appear.

[6] H.T. Ku, M.C. Ku and D.Y. Tang, "Inequalities for eigenvalues of elliptic equations and the generalized. Polya conjecture", Journal of Diff. Equations, 97(1992), 127-139.

[7] H.A. Levine and M.H. Protter, "Unrestricted lower bounds for eigenvalues for classes of elliptic equations and systems of equations with applications to problems in elasticity," Math. Meth. in the Appl. Sci, 7(1985) 210-222.

[8] P. Li and S.T. Yau, "On the Schrödinger equation and the eigenvalue problem," Comm. Math. Phys, 88(1983), 309-318.

[9] L.E. Payne, "New isoperimetric inequalities for eigenvalues and other physical quantities," Comm. Pure Appl. Math, 9, (1956), 531-542.

[10] L.E. Payne, G. Polya and H.F. Weinberger, "On the ratio of consecutive eigenvalues," Journal of Math. and Physics, 35(1956), 289-298.

[11] G. Polya, "On the eigenvalues of vibrating membranes," London Math. Soc., 11(1961), 414-433.

Department of Miathematics and Statistics, University of Massachusetts, Amherst, MA 01003 . U.S.A.

Department of Mathematics and Statistics, University of South Alabama, Mobile, AL 36688. U.S.A. 